\title{
Validation of a FFQ to estimate the intake of PUFA using plasma phospholipid fatty acids and weighed foods records
}

\author{
S. A. McNaughton ${ }^{1,2} *$, M. C. Hughes ${ }^{3}$ and G. C. Marks ${ }^{1}$ \\ ${ }^{1}$ School of Population Health, University of Queensland, Herston Road, Herston, QLD 4006, Australia \\ ${ }^{2}$ Centre for Physical Activity and Nutrition Research, Deakin University, 221 Burwood Highway, \\ Burwood VIC 3125, Australia \\ ${ }^{3}$ Cancer and Population Studies Group, Queensland Institute of Medical Research, 300 Herston Road, Herston, QLD 4006, Australia
}

(Received 11 April 2006 - Revised 23 October 2006 - Accepted 23 October 2006)

\begin{abstract}
Due to the growing knowledge about the role of specific fatty acids in health and disease, dietary intake measurements of individual fatty acids or classes of fatty acids are becoming increasingly important. The objective of this study was to evaluate the ability of the Nambour FFQ to estimate intakes of specific fatty acids, particularly PUFA. The study population was a sub-sample of adult participants in a randomised controlled trial of $\beta$-carotene and sunscreen in the prevention of skin cancer ( $n$ 43). Dietary intake was assessed by a self-administered FFQ and a weighed food record (WFR). Non-fasting blood samples were collected and analysed for plasma phospholipid fatty acids. Median intakes on the FFQ were generally higher than the WFR except for the $n$-3 PUFA groups, where the FFQ estimated higher intakes. Correlations between the FFQ and WFR were moderate ( $r$ 0.32-0.59) except for trans fatty acids ( $r 0.03)$. Correlations between each of the dietary assessment methods and the plasma phospholipids were poor for all fatty acids other than the PUFA. Using the methods of triads approach, the FFQ validity coefficients for total $n-3$ fatty acids, total long chain $n-3$ fatty acids, EPA, arachidonic acid, docosapentaenoic acid and DHA were 0.50, 0.63, 0.45 and 0.62 and 0.62, respectively. For most fatty acids, the FFQ adequately estimates group mean fatty acid intakes and can adequately rank individuals; however, the ability of this FFQ to estimate trans fatty acids was poor.
\end{abstract}

Validation: Fatty acids: Biomarkers: Dietary intake: FFQ: Weighed food record

Current dietary recommendations focus not only on the total fat intake but on the types of fat consumed, in recognition of the importance on differing roles of specific fatty acids in health and disease. Increased $n-3$ intake has been suggested as protective against chronic disease, particularly CVD; however, there is currently ongoing debate about their role in health and further work is required to clarify this (Geleijnse et al. 2006; He \& Song, 2006; Hooper et al. 2006). Trans fatty acids have been associated with the promotion of IHD (Stender \& Dyerberg, 2004). Measurement of dietary intake of specific fatty acids or classes of fatty acids is increasingly important.

While the difficulties associated with the measurement of diet are well recognised, there are a number of issues specific to the measurement of fatty acids. The increasing complexity of the food supply and the availability of a wide range of manufactured and processed foods, coupled with variations in the fats and oils used by the food industry, makes characterisation of the fatty acid composition of foods more difficult (Cantwell, 2000; McNaughton et al. 2004).

Biomarkers of fatty acid status, such as plasma fatty acids, have a number of characteristics useful in the validation of dietary assessment methods. They are often considered objective, as they cannot be altered by the subject and do not rely on a subject's ability to recall intake or on compliance with recording consumption (Kaaks et al. 1997). In addition, they are not susceptible to the same type of measurement errors as other dietary assessment methods, such as errors associated with estimation of portion size or with the use of food composition databases (Gibson, 1990; Neuhoser et al. 2001). However, biomarkers are often not perfectly correlated with dietary intake due to the presence of other determinants, including factors related to metabolism or environmental and lifestyle factors (Hunter, 1998).

Biomarkers of dietary fat intake have been more difficult to identify than for the other nutrients. The ideal biomarker of dietary fat intake would be able to reflect both quantity and quality but currently no marker of total fat intake exists (Arab, 2003). Measurement of individual fatty acids in plasma may be used as biomarkers of fatty acid intake (Ashley, 1996). PUFA have been shown to be the most useful as biomarkers of dietary intake (Arab, 2003). Potential non-dietary determinants of plasma lipids include sex, alcohol and smoking (Simon et al. 1996; Bates et al. 1997).

The objective of this study was to evaluate the ability of the Nambour FFQ to estimate intakes of specific fatty acids, particularly PUFA, using plasma phospholipid measurements and weighed food records (WFR). The validity

Abbreviations: WFR, weighed food record.

* Corresponding author: Dr Sarah McNaughton, fax +6139244 6017, email sarah.mcnaughton@deakin.edu.au 
of the questionnaire with regard to estimates of energy, macronutrients and a range of micronutrients has previously been investigated and published separately (Marks et al. 2006b).

\section{Methods}

A dietary intake validation study was conducted in a randomly selected subset of participants of the Nambour Skin Cancer Study (Ashton et al. 1996; Green et al. 1999; Marks et al. $2006 a, b)$. Only subjects who had already completed the FFQ at baseline in 1992 were eligible for selection. A total of 168 subjects were invited into the study, with 115 subjects agreeing to participate and complete a FFQ and a WFR, representing a response rate of $68.5 \%$.

Demographic variables and health-related behaviours were collected via standardised interviews conducted by trained nurses or self-administered questionnaires (Green et al. 1988). Anthropometric measurements (height and weight) were made during a physical examination coinciding with the administration of the FFQ using standardised protocols (Gordon et al. 1988).

\section{$F F Q$}

Dietary intake was assessed in 1992 by a self-administered, semi-quantitative FFQ based on a questionnaire designed for use in the USA (Willett et al. 1985) and adapted for use in Queensland. The questionnaire consisted of 129 items of specific foods or food groups with nine response options ranging from 'never' to ' $4+$ times per $d$ ' for the frequency of consumption of the specified serving sizes based on household measures (e.g. cups, spoons) and natural units (e.g. 1 slice bread). Among the list of foods specified were low, reduced and full fat dairy products and lean meats $v$. fatty or processed meats. Additional questions on the FFQ collected information on the trimming of visible fat from meat, frequency of fried food at home and fried takeaway food and specific types of fats and oils used on bread, vegetables and in cooking. Questions on the use of self-prescribed nutritional supplements were also included.

Subjects were asked to recall their frequency of consumption of food items over the preceding 6 months. Average daily intake was calculated by expressing the response to the food item as a proportion of daily use, which was then multiplied by the amounts ( $\mathrm{g}$ ) of the specified portion sizes and by the nutrient content of the food. Seasonal foods were weighted according to the proportion of the year that each food was available. Subjects were excluded from analysis if they responded to less than $90 \%$ of items on the FFQ.

\section{Weighed food records}

Subjects completed records on two non-consecutive days, every 2 months for 12 months (during 1993). Starting days were randomly allocated in the initial recording period and then the rest of the week worked through in subsequent recording periods so that weekdays, weekend days and seasons were appropriately weighted over the year. Detailed instructions on the use of the scales and the weighing and recording of food and drink consumed and leftovers were provided for the participants during the initial interview.
Participants were asked to record information on time and place of food and meal preparation, brand names of products and recipes used. For meals eaten away from home, participants were asked to provide detailed descriptions and approximate amounts of what was eaten. Details of any self-prescribed nutritional supplements consumed were also recorded. Data from the WFR were entered into nutritional computer software (Xyris Software, 1991; Xyris Software (Australia) Pty Ltd, Highgate Hill, Queensland, Australia) and the nutrient intakes were then downloaded for subsequent processing and analyses. Subjects were excluded from analysis if they had completed less than $10 \mathrm{~d}$ recording in the WFR.

\section{Plasma phospholipid fatty acids}

A randomly selected subset of subjects had blood samples taken and analysed for plasma phospholipid fatty acids ( $n$ 44). Prior to blood collection, respondents were requested to eat a light breakfast (e.g. cereal, fruit, juice or coffee). Non-fasting venous blood samples of $30 \mathrm{ml}$ were collected using standard venipuncture techniques performed by experienced phlebotomists. Blood samples were processed at the time of collection and serum samples were stored in approximately $1 \mathrm{ml}$ aliquots at $-70^{\circ} \mathrm{C}$ until analysis.

Measurements of plasma phospholipid fatty acids were conducted by Flinders Medical Centre, Adelaide, Australia and are described in detail in Mantzioris et al. (2000). Briefly, plasma was extracted in chloroform:methanol and stored at $-70^{\circ} \mathrm{C}$ before fatty acid analysis. The total lipid extract from the plasma fractions was fractionated by TLC and the phospholipid fractions retained. Fatty acid methyl esters were separated and quantified with gas chromatograph (Hewlett-Packard 6890 with a 50-m capillary column; Hewlett-Packard, Palo Alto, CA, USA). Fatty acid methyl esters were identified based on the retention time to authentic lipid standards (GLC-463; Nuchek Prep Inc. Elysian, MN, USA) and quantified by comparison with the peak area of the internal standard using ChemStation software (Agilent, Palo Alto, CA, USA).

\section{Data analysis}

All analysis in the present study is based on subjects who had completed all three assessments (FFQ, WFR and biomarker; $n$ 44). Fatty acid intakes from the WFR and the FFQ were calculated using a comprehensive fatty acid database for Australian foods (Mann et al. 2003). Energy intakes were assessed to identify subjects with extreme and implausible energy intakes $(>16800 \mathrm{~kJ} / \mathrm{d}$ and $<3360 \mathrm{~kJ} / \mathrm{d}$ for males and $>14700 \mathrm{~kJ} / \mathrm{d}$ and $<2100 \mathrm{~kJ} / \mathrm{d}$ for females; Willett, 1998). One person was excluded on this basis. Fatty acid intakes on the WFR and FFQ represent intakes from food alone (only one subject reported using supplements containing fish oils). Intakes of fatty acids were adjusted for energy intake using the nutrient residual method as recommended in validation studies (Willett, 1998, 2001). Mean intakes on the WFR and FFQ were compared using Student's $t$ tests. Agreement between the FFQ and the WFR was also assessed. The proportions of individuals classified in the same third of the distribution (exact agreement) and into extreme thirds of the distribution (gross misclassification) were calculated. Spearman correlation coefficients were calculated between each of the three dietary 
assessment methods (i.e. FFQ and WFR; FFQ and biomarker; WFR and biomarker) for each fatty acid. Correlations were evaluated as poor $(<0 \cdot 2)$, moderate $(0.2-0.6)$ or good $(>0 \cdot 6)$. For the PUFA, the correlations between each of the three dietary exposure methods were used to calculate the validity coefficient via the method of triads (Ocke \& Kaaks, 1997). The triangular approach to validation assumes that the correlations between the three measurements are explained by the fact that they are all linearly correlated with the true intake. The method of triads provides an upper limit of the validity coefficient while the correlations between the questionnaire measurement and the biomarker measurement provide an estimate of the lower limit of the validity coefficient (Ocke \& Kaaks, 1997; McNaughton et al. 2005). The $95 \%$ CI for the validity coefficients were estimated using bootstrap sampling where 1000 samples of equal size ( $n$ 43) were obtained with replacement from the study subjects. Analyses were performed using SPSS for Windows Version 10.0.5 (SPSS Inc., Chicago, IL, USA). $P$ values $<0.05$ were considered significant.

\section{Results}

Table 1 provides details on the general characteristics for study subjects including age, BMI, smoking status and supplement use. Table 2 presents the mean, standard deviation and median of the dietary intakes of selected fatty acids as measured on the WFR and FFQ and the plasma phospholipid fatty acids. Totals represent the sum of all fatty acids detected in plasma and in foods consumed (not just those listed separately). In general, median intakes on the WFR were higher than the FFQ (up to 1-13-fold higher) except for the $n-3$ PUFA groups, where the FFQ estimated higher intakes (up to 1.40 -fold higher). However, mean intakes were not significantly different between the WFR and FFQ for most of the fatty acids except for total trans fatty acids, with the FFQ providing higher estimates than the WFR. Exact agreement ranged from $30 \%$ (trans fatty acids) to $56 \%$ (oleic acid).
Gross misclassification ranged from $2 \%$ (stearic acid) to $23 \%$ (trans fatty acids).

The Spearman correlation coefficients between each of the three dietary assessment methods are shown in Table 3. The correlations between the FFQ and WFR ranged from 0.32 (linoleic acid and total $n-6$ fatty acids) to 0.59 (total SFA) except for trans fatty acids ( $r$ 0.03). Correlations between each of the dietary assessment methods and the plasma phospholipids were less than 0.20 for all fatty acids other than the PUFA.

With regard to the PUFA, correlations between the WFR and plasma phospholipids ranged from 0.22 to 0.44 for total $n-3$ fatty acids, total long chain fatty acids, arachidonic acid, EPA, docosapentaenoic acid and DHA. Correlations between the FFQ and plasma phospholipids ranged from 0.21 to 0.38 for total $n-3$ fatty acids, total long chain fatty acids, linoleic acid, EPA and DHA. Correlations tended to be stronger between the plasma fatty acids and WFR than the FFQ. Correlations between the plasma phospholipids and both the WFR and FFQ for $\alpha$-linolenic were less than 0.09 while the correlations for plasma phospholipid linoleic acid and total $n-6$ fatty acids showed large variations between the WFR and FFQ. The correlations between the plasma phospholipids and the FFQ and WFR were also calculated when subjects using dietary supplements containing fish oils were excluded (one subject) and results were similar (data not shown). Adjustments for sex, alcohol and smoking did not consistently alter the correlations.

The correlations between each of the three dietary exposure measurements for PUFA were used to calculate the validity coefficient for each of the methods using the method of triads approach along with the $95 \%$ CI calculated by the bootstrap method (Table 4). The range for the validity coefficient is also presented, where the upper limit is that calculated by the method of triads and the lower limit is the correlation between the FFQ and the biomarker. However, when using the method of triads, a validity coefficient cannot be calculated if one of the three correlations is negative (Ocke \& Kaaks,

Table 1. Characteristics of the validation study participants*

(Mean values, standard deviations and range for forty-three participants)

\begin{tabular}{|c|c|c|c|c|c|c|}
\hline & \multicolumn{3}{|c|}{ Females ( $n$ 25) } & \multicolumn{3}{|c|}{ Males $(n 18)$} \\
\hline & Mean & SD & Range & Mean & SD & Range \\
\hline Age (years) & 49.5 & $10 \cdot 2$ & $28-75$ & 53.8 & $12 \cdot 7$ & $29-72$ \\
\hline \multirow[t]{2}{*}{ BMI $\left(\mathrm{kg} / \mathrm{m}^{2}\right)$} & $26 \cdot 3$ & $4 \cdot 0$ & $19 \cdot 6-33 \cdot 7$ & 24.5 & $3 \cdot 6$ & $20 \cdot 0-31 \cdot 1$ \\
\hline & $n$ & $\%$ & & $n$ & $\%$ & \\
\hline \multicolumn{7}{|l|}{ Smoking } \\
\hline Non-smoker & 13 & $54 \cdot 2$ & & 9 & $50 \cdot 0$ & \\
\hline Current smoker & 0 & & & 2 & $11 \cdot 1$ & \\
\hline Ex-smoker & 11 & $45 \cdot 8$ & & 5 & $27 \cdot 8$ & \\
\hline Missing & 1 & 4.0 & & 2 & $11 \cdot 1$ & \\
\hline \multicolumn{7}{|l|}{ Supplement use $†$} \\
\hline Any & 11 & 44 & & 5 & 28 & \\
\hline Containing fish oils & 1 & 1 & & 1 & 1 & \\
\hline
\end{tabular}


Table 2. Plasma phospholipids fatty acids and dietary fatty acid intakes as estimated by weighed food records (WFR) and FFQ $\dagger$ (Mean values, standard deviations and median values for forty-three participants)

\begin{tabular}{|c|c|c|c|c|c|c|c|c|c|c|c|c|}
\hline & & \multicolumn{3}{|c|}{ Plasma $(\mu \mathrm{g} / \mathrm{ml})$} & \multicolumn{3}{|c|}{ FFQ (g) } & \multicolumn{2}{|c|}{ WFR (g) } & \multicolumn{3}{|c|}{ Agreement‡ } \\
\hline & & Mean & SD & Median & Mean & SD & Median & Mean & SD & Median & Exact (\%) & GM (\%) \\
\hline \multirow[t]{3}{*}{ Saturated } & Total & $605 \cdot 56$ & $127 \cdot 85$ & $569 \cdot 12$ & 30.33 & 12.55 & $27 \cdot 22$ & 29.43 & 9.41 & 29.56 & 51 & 7 \\
\hline & Palmitic (16:0) & 362.96 & 82.93 & 346.07 & $15 \cdot 22$ & $5 \cdot 86$ & 13.35 & 14.66 & 4.51 & $14 \cdot 36$ & 40 & 5 \\
\hline & Stearic $(18: 0)$ & 201.35 & $46 \cdot 77$ & $196 \cdot 28$ & 7.31 & $3 \cdot 11$ & 6.62 & 6.95 & $2 \cdot 29$ & 7.02 & 51 & 2 \\
\hline \multirow[t]{2}{*}{ Monounsaturated } & Total & 177.52 & $50 \cdot 81$ & $162 \cdot 82$ & $25 \cdot 40$ & 8.91 & $22 \cdot 50$ & 23.40 & 7.03 & 23.65 & 51 & 12 \\
\hline & Oleic $(18: 1 n-9)$ & 134.56 & 41.47 & $126 \cdot 97$ & 23.39 & $8 \cdot 32$ & 21.01 & 21.66 & 6.56 & $22 \cdot 10$ & 56 & 12 \\
\hline Trans & Total & 13.65 & 3.98 & 13.58 & 0.11 & 0.31 & 0.01 & 0.01 & 0.01 & $0.01^{*}$ & 30 & 23 \\
\hline \multirow[t]{9}{*}{ Polyunsaturated } & Total $n-6$ & 506.06 & $102 \cdot 64$ & $489 \cdot 37$ & 8.47 & 2.93 & 7.84 & $9 \cdot 38$ & 3.78 & 8.86 & 51 & 12 \\
\hline & Total $n-3$ & 79.92 & $21 \cdot 71$ & $78 \cdot 13$ & 1.12 & 0.50 & 1.01 & 1.02 & 0.37 & 0.98 & 44 & 14 \\
\hline & Total long chain $n-3 \S$ & 77.25 & $21 \cdot 25$ & 73.95 & 0.26 & 0.21 & 0.20 & 0.22 & 0.26 & 0.16 & 42 & 12 \\
\hline & Linoleic $(18: 2 n-6)$ & 286.47 & 62.88 & 271.4 & 8.37 & 2.92 & 7.69 & $9 \cdot 28$ & 3.76 & 8.72 & 51 & 12 \\
\hline & Arachidonic acid $(20: 4 n-6)$ & $144 \cdot 85$ & 41.59 & 137.62 & 0.08 & 0.03 & 0.08 & 0.09 & 0.04 & 0.08 & 51 & 7 \\
\hline & $\alpha$-Linolenic acid $(18: 3 n-3)$ & 1.66 & 0.85 & 1.33 & 0.86 & 0.42 & 0.76 & 0.80 & 0.23 & 0.80 & 49 & 9 \\
\hline & $\operatorname{EPA}(20: 5 n-3)$ & 11.46 & 7.49 & 9.39 & 0.09 & 0.08 & 0.07 & 0.08 & 0.11 & 0.05 & 42 & 12 \\
\hline & DPA (22:5n-3) & $15 \cdot 13$ & 4.22 & 14.63 & 0.04 & 0.02 & 0.03 & 0.04 & 0.03 & 0.03 & 42 & 7 \\
\hline & DHA $(22: 6 n-3)$ & 50.67 & $15 \cdot 49$ & 48.85 & 0.12 & $0 \cdot 12$ & 0.09 & 0.11 & 0.13 & 0.08 & 51 & 7 \\
\hline
\end{tabular}

Significantly different mean intakes on the FFQ and WFR ( ${ }^{\star} P<0.05$; two-sided $t$ test). Intakes were log-transformed prior to analysis.

†Energy intakes (kJ) were as follows: FFQ 9969 (SD 2851), median 9598; WFR, 8346 (SD 2246), median 7903.

.

$\S$ Includes EPA, docosapentaenoic acid (DPA) and DHA (Meyer et al. 2003).

For details of subjects and procedures, see p. 562. 
Table 3. Spearman correlation coefficients between each of the three dietary assessment methods $(n 43) \dagger$

\begin{tabular}{|c|c|c|c|c|}
\hline & & FFQ $v$. WFR & WFR v. Biomarker & FFQ v. Biomarker \\
\hline \multirow{3}{*}{ Saturated } & Total & $0.59^{\star}$ & -0.09 & -0.04 \\
\hline & Palmitic (16:0) & $0.46^{*}$ & -0.10 & -0.09 \\
\hline & Stearic $(18: 0)$ & $0.53^{\star}$ & 0.02 & -0.02 \\
\hline \multirow[t]{2}{*}{ Monounsaturated } & Total & $0.48^{*}$ & -0.13 & -0.10 \\
\hline & Oleic $(18: 1 n-9)$ & $0.47^{\star}$ & -0.11 & -0.06 \\
\hline Trans & Total & 0.03 & 0.16 & $0 \cdot 19$ \\
\hline \multirow[t]{9}{*}{ Polyunsaturated } & Total $n-6$ & $0.32^{*}$ & -0.11 & 0.16 \\
\hline & Total $n-3$ & $0.39^{\star}$ & $0.33^{*}$ & 0.21 \\
\hline & Total long chain $n-3$ & $0.46^{\star}$ & $0.44^{\star}$ & $0.38^{\star}$ \\
\hline & Linoleic acid $(18: 2 n-6)$ & $0.32^{*}$ & -0.01 & $0.34^{*}$ \\
\hline & Arachidonic acid $(20: 4 n-6)$ & $0.54^{\star}$ & $0.35^{\star}$ & 0.13 \\
\hline & $\alpha$-linolenic acid $(18: 3 n-3)$ & $0.45^{\star}$ & 0.09 & 0.00 \\
\hline & $\operatorname{EPA}(20: 5 n-3)$ & $0.40^{\star}$ & 0.22 & 0.21 \\
\hline & DPA $(22: 5 n-3)$ & $0.50^{\star}$ & 0.25 & -0.05 \\
\hline & DHA $(22: 6 n-3)$ & $0.52^{\star}$ & $0.43^{*}$ & $0.32^{\star}$ \\
\hline
\end{tabular}

Significant correlations $\left({ }^{*} P<0.05\right)$.

†Fatty acid intakes from the weighed food records (WFR) and FFQ were adjusted for energy intake.

DPA, docosapentaenoic acid.

For details of subjects and procedures, see p. 562.

Table 4. Validity coefficient of the FFQ, weighed food records (WFR) and biomarkers for PUFA as calculated by the method of triads and the $95 \% \mathrm{Cl}$ $(n 43)^{*}$

\begin{tabular}{|c|c|c|c|c|c|c|c|c|c|}
\hline & \multicolumn{6}{|c|}{ Validity coefficients $†$} & \multicolumn{3}{|c|}{ Range of the validity coefficients $\ddagger$} \\
\hline & $\rho_{Q T}$ & $95 \% \mathrm{Cl}$ & $\rho_{\mathrm{BT}}$ & $95 \% \mathrm{Cl}$ & $\rho_{\mathrm{RT}}$ & $95 \% \mathrm{Cl}$ & $\rho_{Q T}$ & $\rho_{\mathrm{BT}}$ & $\rho_{\mathrm{RT}}$ \\
\hline Total $n-3$ & 0.50 & $0.13,1.00$ & 0.43 & $0 \cdot 10,0.81$ & 0.78 & $0.29,1.00$ & $0.21-0.50$ & $0.33-0.43$ & $0.21-0.78$ \\
\hline Total long chain $n-3$ & 0.63 & $0.27,0.98$ & 0.60 & $0.27,0.93$ & 0.73 & $0.34,1.00$ & $0.38-0.63$ & $0.44-0.60$ & $0.38-0.73$ \\
\hline Arachidonic acid $(20: 4 n-6)$ & 0.45 & $0.13,1.00$ & 0.29 & $0.06,0.69$ & 1.00 & $0.43,1.00$ & $0.13-0.45$ & $0.35-0.29$ & $0.13-1.00$ \\
\hline EPA $(20: 5 n-3)$ & 0.62 & $0.18,1.00$ & 0.35 & $0.07,0.78$ & 0.65 & $0.18,1.00$ & $0.21-0.62$ & $0.22-0.35$ & $0.21-0.65$ \\
\hline DHA $(22: 6 n-3)$ & 0.62 & $0.25,0.91$ & 0.52 & $0.16,0.82$ & 0.83 & $0.45,1.00$ & $0.32-0.62$ & $0.43-0.52$ & $0.32-0.83$ \\
\hline
\end{tabular}

* The validity coefficient could not be calculated for total $n-6$ fatty acids, linoleic acid, $\alpha$-linolenic acid and docosapentaenoic acid as one of the three correlations was negative or zero.

†All values $>1.00$ were truncated as this is the highest possible value.

$\ddagger$ The lower limit for the FFQ and the biomarker is the correlation between the FFQ and biomarker, and the lower limit for the WFR is the correlation between the biomarker and the WFR, and the upper limit is calculated by the method of triads.

$\rho_{Q T}$, validity coefficient of the questionnaire; $\rho_{B T}$, validity coefficient of the biomarker; $\rho_{R T}$, validity coefficient of the WFR.

For details of subjects and procedures, see p. 562.

1997); therefore, the validity coefficient could not be calculated for total $n-6$ fatty acids, linoleic acid, $\alpha$-linolenic acid and docosapentaenoic acid. Validity coefficients for the FFQ ranged from 0.45 (arachidonic acid) to 0.63 (total long chain $n-3$ fatty acids). Validity coefficients for the WFR tended to be the highest, followed by the validity coefficients of the FFQ, with those of the plasma biomarkers tending to be the lowest.

\section{Discussion}

The objective of the present study was to evaluate the ability of the Nambour FFQ to estimate intakes of specific fatty acids. When compared with a WFR, mean intakes of individual and specific classes of fatty acids were not significantly different, except for the trans fatty acid intake, where the FFQ provided higher estimates of intake. Correlations between the WFR and FFQ were moderate to good, indicating that the FFQ could adequately rank subjects for most fatty acids. Percentage agreement between the FFQ and WFR showed similar patterns to the correlation analysis, with trans fatty acids showing poor agreement. The magnitude of the correlations between WFR and FFQ in the current study is similar to that seen in other validation studies (Riboli et al. 1997; Brunner et al. 2001) and consistent with previously published results in the larger validity study (Marks et al. 2006b).

Diet-plasma correlations were moderate for total n-3 fatty acids, total long chain fatty acids, linoleic acid, EPA and DHA for both the WFR and the FFQ, although those with the WFR were generally higher. Both methods showed poor correlations for $\alpha$-linolenic acid. As expected due to the capacity for endogenous synthesis of SFA and MUFA, dietplasma correlations were poor for SFA and MUFA. The present results are similar to findings of other studies showing moderate to good correlations for EPA, DHA, linoleic acid ( $r$ 0.3-0.7) and also for all PUFA combined ( $r$ 0.3-0.5; Von Houwelingen et al. 1989; Bonaa et al. 1992; Moilanen et al. 1992; James et al. 1993; Ma et al. 1995; Stallone et al. 1997). Weaker correlations have been shown for SFA and generally poor correlations have been observed for MUFA 
(Moilanen et al. 1992; Ma et al. 1995). Correlations for EPA in the current study were lower than expected when considering the results of previous studies, which have shown equally strong correlations between diet and plasma for DHA and EPA. Correlations between the WFR and biomarker for arachidonic acid were greater than expected given that its metabolism is well regulated (Arab, 2003) and given that Baylin et al. (2005) showed poor correlations $(r 0.05-0 \cdot 12)$ between dietary arachidonic acid measured by FFQ and three biomarkers; results that are consistent with our FFQ results. Kabagambe et al. (2001) used the method of triads approach with adipose tissue fatty acid biomarkers and multiple 24-h dietary recalls and estimated the validity coefficients for a FFQ of 0.59 and 0.89 for $\alpha$-linolenic acid and linoleic acid, respectively. We were not able to estimate validity coefficients for these fatty acids due to negative correlations, so comparisons are not possible.

It is important to note the different reference periods or time frames for each of the dietary exposure measures in the present study. The FFQ was administered in 1992 and asked respondents to consider intake over the previous 6 months. The biomarker measurements were made at approximately the same time and are likely to represent the preceding days to weeks of dietary exposure. In contrast, the WFR were completed approximately 1 year later. The correlations observed may be an underestimate of the true correlations due to the differing time frames.

The use of biomarkers of fatty acid intake is advantageous due to the specific difficulties associated with quantifying the intakes of individual fatty acids and classes of fatty acids. The FFQ included questions relating to trimming of visible fat from meat, frequency of fried food consumption and specific types of fats and oils used, including brand level information for fat spreads and oils, and this information was used in the calculation of fatty acid intakes. Regardless of the level of detail obtained from the subjects, all assessments of nutrient intake are limited by the available food composition database. The availability of a wide range of manufactured and processed foods, coupled with variations in the fats and oils used by the food industry, increases the difficulties associated with the estimation of the fatty acid composition of foods and subsequent estimation of intakes (Mishra et al. 2003; McNaughton et al. 2004). The difficulties associated with incomplete food composition databases have recently been highlighted (McNeill et al. 2004). However, the present study used the most complete fatty acid food composition database currently available for the Australian food supply (Mann et al. 2003).

The current study showed poor correlations for the trans fatty acids. There was a significant decrease in the trans fatty acid content of the food supply in Australia in early 1996 due to the removal of trans fatty acids from many margarines (Clifton et al. 2004). The present study drew on food composition data that would have included data pre and post the decrease in trans fatty acids and we attempted to use the most appropriate data for each food item consumed in the study. The poor correlations observed suggest that this may be a source of error in our estimates of trans fatty acid intake. The poor correlations between $\alpha$-linolenic acid intakes and plasma phospholipid concentrations may have also been affected by errors associated with the food composition data, particularly around trans fatty acids, due to the concurrent changes in PUFA that occur with production of trans fatty acids during partial hydrogenation of vegetables oils (Ascherio \& Willett, 1997).

A variety of factors may affect plasma lipids independent of dietary intake. Smoking and alcohol have been shown to be associated with the concentrations of various fatty acids independently of dietary fat, energy, cholesterol and BMI in previous studies, which suggested that smoking and alcohol intake may impact on the absorption, synthesis or metabolism of fatty acids (Simon et al. 1996). In the current study, alcohol and smoking were not associated with plasma fatty acid levels (data not shown) and adjustment of the diet-plasma correlations for alcohol and smoking had no consistent effects.

Previous work in this study population (Marks et al. $2006 a, b$ ) has shown that the validity of the FFQ varies according to a number of personal factors or subject characteristics. For example, with regard to fat intakes, age, BMI, sex and presence of a medical condition were shown to be independent predictors of the difference in intakes on the FFQ and WFR. These factors will presumably act in a similar way with regard to individual fatty acids; however, the small sample size of the current study precluded their investigation. Therefore, analyses of diet-disease relationships investigating individual fatty acid intakes would require adjustment for these factors as previously suggested for the major fat sub-groups (Marks et al. 2006b).

The method of triads has been applied in a small number of dietary validation studies and is used to determine the validity coefficient of a questionnaire measure of intake using two additional measures, such as WFR and biomarkers (Kaaks et al. 1997; Ocke \& Kaaks, 1997; Kabagambe et al. 2001; Fowke et al. 2002; Pufulete et al. 2002; Bhakta et al. 2005; Brevik et al. 2005; Shai et al. 2005). The triangular approach to validation uses the correlations between each of the three methods to calculate the validity coefficient. It assumes that the correlations between the three measurements are explained by the fact that they are all linearly correlated with the true intake and assumes that errors associated with each of the methods are not correlated. As the FFQ and WFR may have some common sources of error (e.g. food composition database values) and therefore their errors may be correlated, so the validity coefficient provided by the method of triads should be considered an upper limit. A lower limit for the validity coefficient is provided by the correlations between the questionnaire measurement and the biomarker measurement. The method of triads approach does have some limitations, for example, the occurrence of values $>1$, known as Heywood cases, which may result from either random sampling variations or violations to the model assumptions (Ocke \& Kaaks, 1997; Daures et al. 2000). Also, its use is restricted to situations in which a biomarker that is known to reflect to diet is available (Shai et al. 2005). Hence, the use of the method of triads was restricted in the present study to measures of PUFA intake.

Reproducibility and validity studies are generally conducted in a sub-sample of a population being investigated as part of a larger epidemiological investigation. Differences between the sample used for the validity study and the overall study population with regard to education level, sex and age may influence the validity of the dietary assessment tool (Armstrong et al. 
1992). Similarly, differences in the distribution of the dietary exposure between the validity study group and the overall study group may affect the validity of the method (Armstrong et al. 1992). In addition, subjects who participate in dietary validation studies may be different from the general population due to their willingness to participate and compliance with recording dietary intake and this may result in overestimation of the validity of the FFQ (Armstrong et al. 1992). Subjects who participated in the current validation study and had dietary exposure data available from all three sources were similar to the overall Nambour Skin Cancer Study group with regard to a number of factors, including age, BMI, smoking status and the proportion of males and females present (Green et al. 1999). With regard to dietary and plasma fatty acids, intakes of fatty acids in this sample were comparable with intakes of the general Australian population based on the 1995 Australian National Nutrition Survey, an analysis using the same food composition database (Meyer et al. 2003) and plasma phospholipid concentrations were also similar to other studies of Australian adults (Mantzioris et al. 2000; Woods et al. 2002).

The present study evaluated the ability of the Nambour Skin Cancer Study FFQ to estimate PUFA intakes. Using methods of triads approach we determined that validity coefficients for the FFQ were greater than 0.40 for the PUFA. For SFA and MUFA, comparisons of the FFQ and the WFR suggest that the FFQ adequately estimates group mean fatty acid intakes and can adequately rank individuals. However, trans fatty acids were poorly estimated by this particular FFQ.

\section{Acknowledgements}

The study was supported by the Public Health Research and Development Committee of the National Health and Medical Research Council (NHMRC) of Australia and the World Cancer Research Fund International. Sarah McNaughton is supported by a NHMRC Public Health Research Fellowship (ID 323519).

\section{References}

Arab L (2003) Biomarkers of fat and fatty acid intake. J Nutr 133, 925S-932S.

Armstrong BK, White E \& Saracci R (1992) Principles of Exposure Measurement in Epidemiology. New York: Oxford University Press.

Ascherio A \& Willett WC (1997) Health effects of trans fatty acids. Am J Clin Nutr 66, 1006S-1010S.

Ashley JM (1996) Lipid biomarkers of adherence to low fat diets. Adv Exp Med Biol 399, 115-129.

Ashton BA, Marks GC, Battistutta D, Green AC \& the Nambour Study Group (1996) Under-reporting of energy intake in two methods of dietary assessment in the Nambour Trial. Aust J Nutr Diet 53, 53-60.

Bates CJ, Thurnham DI, Bingham SA, Margetts BM \& Nelson M (1997) Biochemical markers of nutrient intake. In Design Concepts in Nutritional Epidemiology, pp. 170-240 [BM Margetts and M Nelson, editors]. New York: Oxford University Press.

Baylin A, Kim MK, Donovan-Palmer A, Siles X, Dougherty L, Tocco P \& Campos H (2005) Fasting whole blood as a biomarker of essential fatty acid intake in epidemiologic studies: comparison with adipose tissue and plasma. Am J Epidemiol 162, 373-381.

Bhakta D, Dos Santos Silva I, Higgins C, Sevak L, KassamKhamis T, Mangtani P, Adlercreutz H \& McMichael A (2005)
A semiquantitative food frequency questionnaire is a valid indicator of the usual intake of phytoestrogens by south Asian women in the UK relative to multiple 24-h dietary recalls and multiple plasma samples. $J$ Nutr 135, 116-123.

Bonaa KH, Bjerve KS \& Nordoy A (1992) Habitual fish consumption, plasma phospholipid fatty acids, and serum lipids the Tromso study. Am J Clin Nutr 55, 1126-1134.

Brevik A, Veierod MB, Drevon CA \& Andersen LF (2005) Evaluation of the odd fatty acids 15:0 and 17:0 in serum and adipose tissue as markers of intake of milk and dairy fat. Eur J Clin Nutr 59, 1417-1422.

Brunner E, Stallone D, Juneja M, Bingham S \& Marmot M (2001) Dietary assessment in Whitehall II: Comparison of $7 \mathrm{~d}$ diet diary and food-frequency questionnaire and validity against biomarkers. Br J Nutr 86, 405-414.

Cantwell MM (2000) Assessment of individual fatty acid intake. Proc Nutr Soc 59, 187-191.

Clifton PM, Keogh JB \& Noakes M (2004) Trans fatty acids in adipose tissue and the food supply are associated with myocardial infarction. $J$ Nutr 134, 874-879.

Daures JP, Gerber M, Scali J, Asre C, Bonifacj C \& Kaaks R (2000) Validation of a food-frequency questionnaire using multiple-day records and biochemical markers: application of the triads method. J Epidemiol Biostat 5, 109-115.

Fowke JH, Hebert JR \& Fahey JW (2002) Urinary excretion of dithiocarbamates and self-reported Cruciferous vegetable intake: application of the 'method of triads' to a food-specific biomarker. Public Health Nutr 5, 791-799.

Geleijnse JM, Brouwer IA \& Feskens EJ (2006) Risks and benefits of omega 3 fats: health benefits of omega 3 fats are in doubt. Br Med $J$ 332, 915.

Gibson RS (1990) Principles of Nutritional Assessment. New York: Oxford University Press.

Gordon CC, Chumlea WC \& Roche AF (1988) Stature, recumbent length and weight. In Anthropometric Standardization Reference Manual. Abridged Edition, pp. 3-8 [TG Lohman, AF Roche and R Martorell, editors]. Champaign, Illinois: Human Kinetic Books. Green A, Beardmore G, Hart V, Leslie D, Marks R \& Staines D (1988) Skin cancer in a Queensland population. J Am Acad Dermatol 19, 1045-1052.

Green A, Williams G, Neale R, et al. (1999) Daily sunscreen application and betacarotene supplementation in prevention of basalcell and squamous-cell carcinomas of the skin: a randomised trial. Lancet 354, 723-729.

He K \& Song Y (2006) Risks and benefits of omega 3 fats: a few thoughts on systematic review. Br Med J 332, 915.

Hooper L, Thompson RL, Harrison RA, et al. (2006) Risks and benefits of omega 3 fats for mortality, cardiovascular disease, and cancer: systematic review. Br Med J 332, 752-760.

Hunter D (1998) Biochemical indicators of dietary intake. In Nutritional Epidemiology, pp. 174-243 [WC Willett, editor]. New York: Oxford University Press.

James MJ, Gibson RA, D’Angelo M, Neumann MA \& Cleland LG (1993) Simple relationships exist between dietary linoleate and the $\mathrm{n}-6$ fatty acids of human neutrophils and plasma. Am J Clin Nutr 58, 497-500.

Kaaks R, Riboli E \& Sinha R (1997) Biochemical markers of dietary intake. In Application of Biomarkers in Cancer Epidemiology, pp. 103-126 [P Toniolo, P Boffetta, DEG Shuker, N Rothman, B Hulka and N Pearce, editors]. Lyon: International Agency for Research on Cancer.

Kabagambe EK, Baylin A, Allan DA, Siles X, Spiegelman D \& Campos $\mathrm{H}$ (2001) Application of the method of triads to evaluate the performance of food frequency questionnaires and biomarkers as indicators of long-term dietary intake. Am J Epidemiol 154, 1126-1135.

Ma J, Folsom AR, Shahar E \& Eckfeldt JH The Atherosclerosis Risk in Communities Study Investigators (1995) Plasma fatty acid 
composition as an indicator of habitual dietary fat intake in middleaged adults. Am J Clin Nutr 62, 564-571.

McNaughton SA, Marks GC, Gaffney P, Williams G \& Green A (2005) Validation of a food frequency questionnaire assessment of carotenoid and vitamin E intake using weighed food records and plasma biomarkers: The method of triads model. Eur J Clin Nutr 59, 211-218.

McNaughton SA, Moore CS, Bryant SP, Matthews N, Paul AA \& Jebb SA (2004) Relationship between dietary intake of essential polyunsaturated fatty acids and plasma status in a food-based intervention study. Proc Nutr Soc 63, 38A.

McNeill G, Peace H \& Masson F (2004) Beware the fatty acid free sausage. J Hum Nutr Diet 17, 71-72.

Mann NJ, Sinclair AJ, Percival P, Lewis JL, Meyer BJ \& Howe PRC (2003) Development of a database of fatty acids in Australian foods. Nutr Diet 60, 42-45.

Mantzioris E, Cleland LG, Gibson RA, Neumann MA, Demasi M \& James MJ (2000) Biochemical effects of a diet containing foods enriched with n-3 fatty acids. Am J Clin Nutr 72, 42-48.

Marks GC, Hughes M \& Van der Pols JC (2006a) Relative validity of food intake estimates using a food frequency questionnaire is associated with sex, age, and other personal characteristics. J Nutr 136, 459-465.

Marks GC, Hughes MC \& Van der Pols JC (2006b) The effect of personal characteristics on the validity of nutrient intake estimates using a food frequency questionnaire. Public Health Nutr 9, 394-402.

Meyer BJ, Mann NJ, Lewis JL, Milligan GC, Sinclair AJ \& Howe PRC (2003) Dietary intake and food sources of omega-6 and omega-3 polyunsaturated fatty acids. Lipids 38, 391-398.

Mishra GD, Malik NS, Paul AA, Wadsworth ME \& Bolton-Smith C (2003) Childhood and adult dietary vitamin E intake and cardiovascular risk factors in mid-life in the 1946 British Birth Cohort. Eur J Clin Nutr 57, 1418-1425.

Moilanen T, Rasanen L, Viikari J, Akerblom HK \& Nikkari T (1992) Correlation of serum fatty acid composition with dietary intake data in children and young adults. Ann Med 24, 67-70.

Neuhoser ML, Patterson RE, Kristal AR, Eldridge AL \& Vizenor NC (2001) A brief dietary assessment instrument for assessing target foods, nutrients and eating patterns. Public Health Nutr 4, 73-78.

Ocke MC \& Kaaks RJ (1997) Biochemical markers as additional measurements in dietary validity studies: application of the method of triads with examples from the European Prospective Investigation into Cancer and Nutrition. Am J Clin Nutr 65, 1240S-1245S.

Pufulete M, Emery PW, Nelson M \& Sanders TAB (2002) Validation of a short food frequency questionnaire to assess folate intake. $\mathrm{Br} \mathrm{J}$ Nutr 87, 383-390.

Riboli E, Elmstahl S, Saracci R, Gullberg B \& Lindgarde F (1997) The Malmo Food Study: validity of two dietary assessment methods for measuring nutrient intake. Int $J$ Epidemiol 26, S161-S173.

Shai I, Rosner BA, Shahar DR, Vardi H, Azrad AB, Kanfi A, Schwarzfuchs D \& Fraser D (2005) Dietary evaluation and attenuation of relative risk: multiple comparisons between blood and urinary biomarkers, food frequency, and 24-hour recall questionnaires: the DEARR study. J Nutr 135, 573-579.

Simon JA, Fong J, Bernert JT \& Browner WS (1996) Relation of smoking and alcohol consumption to serum fatty acids. Am J Epidemiol 144, 325-334.

Stallone DD, Brunner EJ, Bingham SA \& Marmot MG (1997) Dietary assessment in Whitehall II the influence of reporting bias on apparent socioeconomic variation in nutrient intakes. Eur J Clin Nutr 51, 815-825.

Stender S \& Dyerberg J (2004) Influence of trans fatty acids on health. Ann Nutr Metab 48, 61-66.

Von Houwelingen AC, Kester AD, Kromhout D \& Hornstra G (1989) Comparison between habitual intake of polyunsaturated fatty acids and their concentrations in serum lipid fractions. Eur J Clin Nutr 43, 11-20.

Willett W (1998) Nutritional Epidemiology. New York: Oxford University Press.

Willett W (2001) Dietary diaries versus food frequency questionnaires - a case of undigestible data. Int $J$ Epidemiol 30, 317-319.

Willett WC, Sampson L, Stampfer MJ, Rosner B, Bain C, Witschi J, Hennekens CH \& Speizer FE (1985) Reproducibility and validity of a semiquantitative food frequency questionnaire. Am J Epidemiol 122, 51-65.

Woods RK, Stoney RM, Ireland PD, Bailey MJ, Raven JM, Thien FCK, Walters DE \& Abramson MJ (2002) A valid food frequency questionnaire for measuring dietary fish intake. Asia Pac J Clin Nutr 11, 56-61. 